\title{
Low-complexity Joint Sub-carrier Phase Noise Compensation for Digital Multi-carrier Systems
}

Yankov, Metodi Plamenov; Barletta, Luca; Zibar, Darko

Published in:

Proceedings of European Conference on Optical Communications

Link to article, DOI:

10.1109/ECOC.2017.8345922

Publication date:

2017

Document Version

Peer reviewed version

Link back to DTU Orbit

Citation (APA):

Yankov, M. P., Barletta, L., \& Zibar, D. (2017). Low-complexity Joint Sub-carrier Phase Noise Compensation for Digital Multi-carrier Systems. In Proceedings of European Conference on Optical Communications [p.

P1.SC3.39] IEEE. https://doi.org/10.1109/ECOC.2017.8345922

\section{General rights}

Copyright and moral rights for the publications made accessible in the public portal are retained by the authors and/or other copyright owners and it is a condition of accessing publications that users recognise and abide by the legal requirements associated with these rights.

- Users may download and print one copy of any publication from the public portal for the purpose of private study or research.

- You may not further distribute the material or use it for any profit-making activity or commercial gain

- You may freely distribute the URL identifying the publication in the public portal

If you believe that this document breaches copyright please contact us providing details, and we will remove access to the work immediately and investigate your claim 


\title{
Low-complexity Joint Sub-carrier Phase Noise Compensation for Digital Multi-carrier Systems
}

\author{
Metodi P. Yankov ${ }^{(1)}$, Luca Barletta ${ }^{(2)}$, Darko Zibar ${ }^{(1)}$ \\ (1) Department of Photonics Engineering, Technical University of Denmark, 2800 Kgs. Lyngby, Denmark \\ meya@fotonik.dtu.dk \\ (2) Department of Electronics Information and Bioengineering, Politecnico di Milano, 20133 Milan, Italy
}

Abstract Joint sub-carrier phase noise processing is proposed which recovers the SNR penalty related to decreased sub-carrier baudrate w.r.t. single carrier systems. The method enables digital sub-banding to be safely employed for nonlinear mitigation for modulation formats of up to 256-QAM.

\section{Introduction}

Digital sub-carrier modulation (SCM) has recently attracted significant attention due to its resilience to nonlinearities in wavelength division multiplexed (WDM) optical fiber systems. Theoretical predictions with the enhanced Gaussian noise model ${ }^{1}$ show that the symbol rate per channel can be optimized for maximum transmission reach to between $2 \mathrm{GBd}$ and $10 \mathrm{GBd}$, also confirmed experimentally for up to 16-quadrature amplitude modulation (QAM) formats ${ }^{2,3}$. Recently, it was suggested that the gains may be significant also for higher-order modulation formats, e.g. 64QAM and 256-QAM assuming nonlinear phase noise (NLPN) compensation is performed ${ }^{4}$.

The standard receiver architecture for multicarrier (MC) systems performs independent phase noise processing of each sub-carrier, e.g. with the simple Viterbi \& Viterbi algorithm ${ }^{2}$, which is known to be sufficient for QPSK signals. However, for larger modulation formats, more sophisticated methods are needed to study the nonlinear tolerance of SCM. Alternatively, data-aided approaches are required ${ }^{3}$. Carrier phase recovery is particularly problematic for MC systems, where the symbol rate per sub-carrier is smaller and each sub-carrier is thus more affected by local oscillator (LO) phase noise. Joint carrier phase recovery was proposed in ${ }^{5}$ for 16 -QAM modulation. Higher order modulations are covered with the pilot-aided algorithm ${ }^{6}$, later extended for multicarrier processing ${ }^{7}$.

In this paper, digital SCM with high-order constellations (up to 256-QAM) is treated, where a single optical chain is employed at the transmitter and receiver. An extension of the algorithm from ${ }^{8}$ is proposed for joint sub-carrier phase recovery. The proposed algorithm is shown to recover almost entirely the penalty of SCM related to the

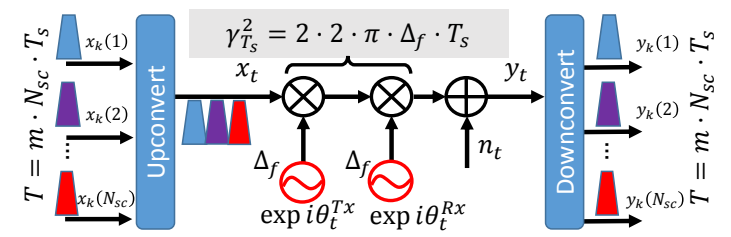

Fig. 1: A block diagram of the model for the considered multi-channel transmission.

above-mentioned reduced sub-carrier baudrate.

\section{Channel model}

A standard phase noise channel is considered, where the LO and transmitter laser are modeled as Wiener processes. The channel is of the form

$$
y_{t}=x_{t} \cdot e^{j \phi_{t}}+n_{t}
$$

where $y_{t}$ and $x_{t}$ are the channel output and input, respectively, $n_{t}$ are additive, white Gaussian noise (AWGN) samples, $\phi_{t}$ are the phase noise samples at times $t \cdot T_{s}$ and $T_{s}$ is the sampling period (for justification of the model see ${ }^{8}$ ). The samples $\phi_{t}$ model the combined effect of transmitter laser and LO, and evolve as

$$
\phi_{t}=\phi_{t-1}+2 \cdot 2 \pi \Delta_{f} T_{s},
$$

where $\Delta_{f}$ is the laser linewidth (assumed equal at transmitter and receiver). The process noise variance is defined as $\gamma_{T_{s}}^{2}=2 \cdot 2 \pi \Delta_{f} T_{s}$.

A block diagram of the considered system is given in Fig. 1. At the transmitter, multiple subcarriers are combined by up-sampling and pulse shaping. The samples $x_{k}$ in each sub-carrier at time $k \cdot T$ are drawn from a finite-size constellation $\mathcal{X}$, in this paper QAM. The symbol period is $T=m \cdot N_{s c} \cdot T_{s}$, where $m$ is the oversampling factor and $N_{s c}$ is the number of sub-carriers. At the receiver, the sub-carriers are down-converted, down-sampled to 1 sample per symbol, and sent for processing. The equivalent channel for $N_{s c}^{J P}$ 
jointly processed sub-carriers can be modeled as a diagonal multiple-input multiple-output (MIMO) channel of the form

$$
\mathbf{y}_{k}=\mathbf{x}_{k} \cdot e^{j \theta_{k}}+\mathbf{n}_{k},
$$

where $\mathbf{y}_{k}, \mathbf{x}_{k}$ and $\mathbf{n}_{k}$ are vectors of length $N_{s c}^{J P}$. Similar to the process $\left\{\phi_{t}\right\}$, the phase noise $\left\{\theta_{k}\right\}$ is modeled as a Wiener process with process noise variance $\gamma_{T}^{2}=2 \cdot 2 \pi \Delta_{f} T$.

\section{Joint sub-carrier phase noise compensation}

$\mathrm{In}^{8}$, a low-complexity pilot-based method was proposed for phase noise processing, which is able to operate at a low SNR relative to the modulation format size, e.g. at the $25 \%$ and higher overhead FEC limit. The method, referred to as the Tikhonov Mixture Model (TMM), was shown to outperform standard decision-directed methods, which are penalized due to the increased symbol error rate at that operating point where the uncoded BER is $\approx 10^{-1}$. The method estimates the posterior distributions of the transmitted symbols (also referred to as the posteriors for brevity) $p\left(\mathbf{x}_{k} \mid \mathbf{y}_{1}^{K}\right)$, which are used both for achievable information rate (AIR) estimation, but also for the sub-sequent demodulation. Here $(\cdot)_{1}^{K}$ represents the sequence from time 1 to $K$. The posteriors are estimated by forward and backward recursions on a factor graph, where the forward message $p\left(\theta_{k} \mid \mathbf{y}_{1}^{k-1}\right)$ and backward message $p\left(\mathbf{y}_{k+1}^{K} \mid \theta_{k}\right)$ are modeled as mixtures of Tikhonov distributions in $\theta_{k}$

$$
\begin{array}{r}
p\left(\theta_{k} \mid \mathbf{y}_{1}^{k-1}\right)=\sum_{m=1}^{M} \bar{\alpha}_{m, k} t\left(\bar{w}_{m, k} ; \theta_{k}\right), \\
p\left(\mathbf{y}_{k+1}^{K} \mid \theta_{k}\right)=\sum_{n=1}^{N} \bar{\beta}_{n, k} t\left(\bar{u}_{n, k} ; \theta_{k}\right) .
\end{array}
$$

In (4), $\bar{\alpha}_{m, k}$ and $\bar{\beta}_{n, k}$ are mixing coefficients, $t\left(w ; \theta_{k}\right)$ is the Tikhonov distributions of variable $\theta_{k}$ with complex parameter $w$, and $M$ and $N$ are the number of components in the forward and backward recursions, respectively. Joint sub-carrier processing requires replacing the scalar math in the recursions by vector math. The graph messages remain Tikhonov mixtures in $\theta_{k}$, however, the mixture parameters $\bar{w}_{m, k}$ and $\bar{u}_{n, k}$ are estimated for a single phase noise process but from multiple sources jointly, leading to an improved performance. The complete derivations of (4) updated from ${ }^{8}$ to vector math are omitted for brevity. Under the model (3), the channel likelihood factorizes as $p\left(\mathbf{y}_{k} \mid \mathbf{x}_{k}, \theta_{k}\right)=\prod_{i} p\left(y_{k}(i) \mid x_{k}(i), \theta_{k}\right)$, where $x_{k}(i)$ and $y_{k}(i)$ are the input and output symbols at time $k$ on the $i$-th sub-carrier, respectively. Assuming the data on different sub-carriers are independent, the final posteriors can then be expressed as

$$
\begin{array}{r}
p\left(\mathbf{x}_{k} \mid \mathbf{y}_{1}^{K}\right)=p\left(\mathbf{x}_{k}\right) \sum_{m=1}^{M} \bar{\alpha}_{m, k} \sum_{n=1}^{N} \bar{\beta}_{n, k} \times \\
\int_{-\pi}^{\pi} p\left(\mathbf{y}_{k} \mid \mathbf{x}_{k}, \theta_{k}\right) t\left(\bar{u}_{n, k} ; \theta_{k}\right) t\left(\bar{w}_{m, k} ; \theta_{k}\right) d \theta_{k} \\
=\sum_{m=1}^{M} \bar{\alpha}_{m, k} \sum_{n=1}^{N} \bar{\beta}_{n, k} \prod_{i} p\left(x_{k}(i)\right) \times \\
\int_{-\pi}^{\pi} p\left(y_{k}(i) \mid x_{k}(i), \theta_{k}\right) t\left(\bar{u}_{n, k} ; \theta_{k}\right) t\left(\bar{w}_{m, k} ; \theta_{k}\right) d \theta_{k} .
\end{array}
$$

The last integral above can be solved in closed form similarly to the single carrier case ${ }^{8}$. As discussed, the TMM algorithm has lower complexity than standard decision directed methods. The proposed multi-carrier algorithm requires the same amount of computations as the standard algorithm applied to each sub-carrier independently, and thus there is no added complexity, which scales linearly with $N_{s c}^{J P}$.

\section{Results}

The proposed method is studied in a standard, single mode fiber $(\alpha=0.2 \mathrm{~dB} / \mathrm{km}, D=17 \mathrm{ps} /(\mathrm{nm}$. $\mathrm{km}), \gamma=1.3(\mathrm{~W} \cdot \mathrm{km})^{-1}$ ) transmission simulated with the split-step Fourier method. Lumped amplification with erbium doped fiber amplifiers (noise figure of $5 \mathrm{~dB}$ ) is considered with span length of $100 \mathrm{~km}$. For simplicity, single polarization WDM system is simulated, in which each of the three simulated WDM channels has $56 \mathrm{GHz}$ bandwidth divided into $N_{s c}$ sub-carriers. It was verified in a linear channel with an ideal laser scenario that the 0.001 roll-off factor of the pulse-shaping filters with $50 \mathrm{MHz}$ guardband does not result in measurable linear inter-sub-carrier interference. The central channel is evaluated in terms of its AIR, averaged over all sub-carriers. The AIR represents the maximum data rate which can be communicated reliably through the channel and is measured in bits/QAM symbol in this paper.

The pilot symbols are spread through the subcarrier sequences as uniformly as possible as shown in Fig. 2. The pilots are used by replacing the prior probability with $p\left(x_{k}(i)\right)=1$ for the

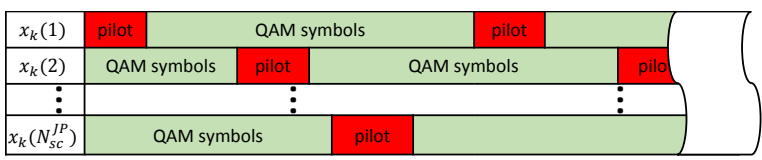

Fig. 2: Pilot spreading illustration. 

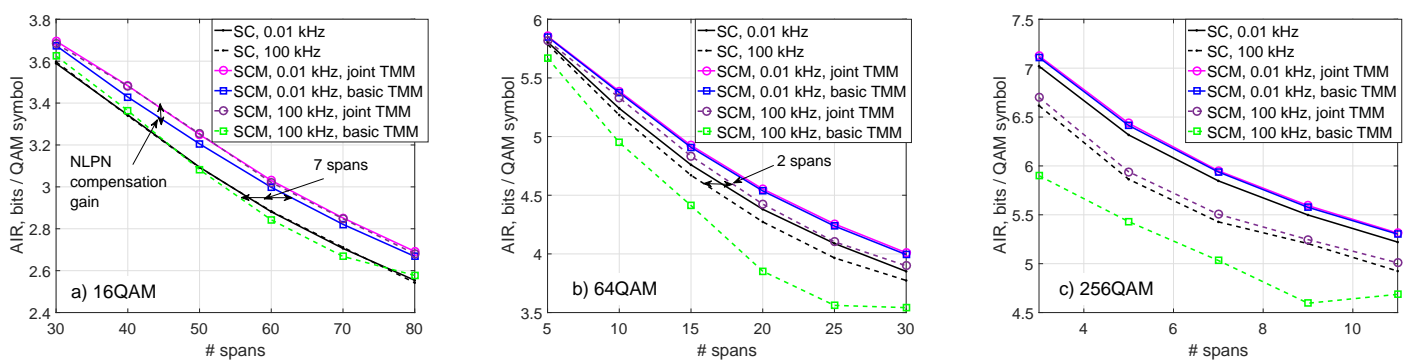

Fig. 3: Performance for WDM transmission of SCM and single carrier (SC) systems. Nonlinear gain is achieved for all modulation formats - $10 \%$ for 256 -QAM and increased to $15 \%$ for 16 -QAM, independently of the laser linewidth. The proposed method achieves additional NLPN compensation gain.

true transmitted symbol on the $i$-th sub-carrier at pilot symbol time $k$, and with $p\left(x_{k}(i)\right)=0$ for the rest of the symbols in the alphabet. For all other symbol positions, $p\left(x_{k}(i)\right)=1 /|\mathcal{X}|$. The pilot rate was optimized to $0.5 \%$ and is taken into account when estimating the AIR. Higher pilot rate allows for improved phase noise estimation and tracking, however, it results in increased loss in maximum AIR, and ultimately worse performance. For comparison, $\mathrm{in}^{6}, 2 \%$ pilot rate is used for 256-QAM, which is not taken into account.

In Fig. 3, the AIR is given as a function of the distance for 16, 64 and 256-QAM at the optimal total launch power $(\approx 4 \mathrm{dBm}$ and $\approx 5 \mathrm{dBm}$ for single carrier (SC) and SCM systems, respectively). The number of sub-carriers was optimized to $N_{s c}=13$ at 50 spans and 20 spans for 16-QAM and 64-QAM, respectively, and to $N_{s c}=5$ at 5 spans for 256-QAM. In the case of insignificant laser phase noise $\left(\Delta_{f}=10 \mathrm{~Hz}\right.$, solid lines), for 16-QAM, between 5 and 10 spans, corresponding to between $10 \%$ and $15 \%$ can be gained at short and long distance, respectively. The gain is slightly increased with joint phase noise processing. We attribute this additional gain to improved NLPN compensation, which was also previously demonstrated for the basic TMM algorithm $^{9}$. The additional gain for joint processing also suggests that the NLPN is correlated across sub-carriers, which is exploited with the proposed joint TMM algorithm. The gains are preserved when non-ideal lasers are employed $\left(\Delta_{f}=100\right.$ $\mathrm{kHz}$, dashed lines) regardless of the target AIR, whereas for independent sub-carrier processing, the nonlinear tolerance gains of SCM are masked by the loss, originating in poorer laser phase noise compensation. Joint phase noise processing enables around 2 spans of gain for 64-QAM and less than a span for 256-QAM regardless of the laser linewidth. The gains are generally smaller than the 16-QAM gains from Fig. 3(a) due to the shorter distance and correspond to $\approx 10 \%$ of reach.

\section{Conclusions}

An algorithm was proposed for joint sub-carrier phase noise compensation operating at no additional complexity and insignificant penalty due to the reduced sub-carrier baudrate. The proposed algorithm allows for digital sub-carrier modulation to be safely employed for non-linear noise mitigation in WDM systems operating with high-order QAM.

\section{Acknowledgements}

This work was supported by the DNRF Research Centre of Excellence, SPOC, ref. DNRF123.

\section{References}

[1] P. Poggiolini et al., "Analytical results on system maximum reach increase through symbol rate optimization," Proc. OFC, Th.3.D.6., (2015).

[2] P. Poggiolini et al., "Analytical and experimental results on system maximum reach increase through symbol rate optimization," JLT, 34(8), p. 1872 (2016).

[3] F. P. Guiomar et al., "Nonlinear mitigation on subcarriermultiplexed PM-16QAM optical systems," Opt. Exp., 25(4), p. 4298 (2017).

[4] R. Dar and P. J. Winzer, "Nonlinear interference mitigation: methods and potential gain," JLT, 35(4), p. 903 (2017).

[5] M. Qiu et al., "Laser phase noise effects and joint carrier phase recovery in coherent optical transmissions with digital subcarrier multiplexing," IEEE Photonics Journal, 9(1), p. 7901013, (2017).

[6] M. Pajovic et al., "Experimental demonstration of multipilot aided carrier phase estimation for DP-64QAM and DP-256QAM," Proc. ECOC, Mo.4.3.3, (2005).

[7] D. S. Millar et al., "Design of a $1 \mathrm{~Tb} / \mathrm{s}$ superchannel coherent receiver," JLT, 34(6), p. 1453 (2016).

[8] M. P. Yankov et al., "Low-complexity tracking of laser and nonlinear phase noise in WDM optical fiber systems," JLT, 33(23), p. 4975 (2015).

[9] M. P. Yankov et al., "Nonlinear phase noise compensation in experimental WDM systems with 256QAM," JLT, 35(8), p. $1438(2017)$ 